\title{
Spububsaúde
}

\section{Aspectos relacionados a associação entre obesidade e Covid- 19: uma revisão integrativa}

\author{
Aspects relating to the association between obesity and Covid-19: an integrative review
}

\author{
Bruna Leite Lourenço $^{1} \bullet$, Fabiana Maria Rodrigues Lopes de Oliveira $^{2} \bullet$, Mariana Guedes Almeida ${ }^{3} \bullet$, \\ Renata de Aquino Ferreira ${ }^{1 *} \bullet$, Sarah Emily Ramos de Sousa ${ }^{2} \bullet$
}

\begin{abstract}
${ }^{1}$ Medicina, Centro Universitário de João Pessoa, João Pessoa, Paraíba, Brasil. ${ }^{2}$ Enfermagem, Centro Universitário de João Pessoa, João Pessoa, Paraíba, Brasil. ${ }^{3}$ Fisioterapia, Centro Universitário de João Pessoa, João Pessoa, Paraíba, Brasil. *Autor correspondente. E-mail: referreira012@gmail.com
\end{abstract}

\begin{abstract}
Resumo: Introdução: No final do ano de 2019, foi identificado um novo tipo de coronavírus, nomeado de SARS-CoV-2 o qual se descobriu ser responsável pela doença COVID-19. Alguns pacientes desenvolvem a forma grave da doença, sendo necessários cuidados intensivos, a exemplo de pessoas com comorbidades. Dentre essas comorbidades, destaca-se a obesidade, ressaltando assim a importância de conhecer os aspectos relacionados à infecção pelo vírus, sobretudo sua associação com esse agravo à saúde. Revisão: trata-se de uma revisão integrativa da literatura, sendo utilizadas bases de dados como a LILACS, MEDLINE, SciELO e PUBMED pesquisada através das combinações dos seguintes descritores em Ciências da Saúde (DeCS) e seus correspondentes no Medical Subject Headings (Mesh): coronavirus infections, obesity, comorbidity e risk factors. Discussão: 32 artigos foram analisados à luz da literatura, demonstrando a relação entre obesidade e a infecção pelo SARS-CoV-2. Considerações finais: Com os achados do estudo foi possível compreender que a obesidade se apresenta como um fator de risco para a COVID-19 grave, esses fatores implicam em complicações e repercussões importantes da pessoa com obesidade: ventilação mecânica, hospitalização e cuidados intensivos
\end{abstract}

Palavras-chaves: comorbidade, fatores de risco, infecções por coronavírus, obesidade.

\begin{abstract}
Introduction: At the end of 2019, a new type of coronavirus was identified, named SARS-CoV-2 which was found to be responsible for the disease COVID-19. Some patients develop the severe form of the disease, requiring intensive care, such as people with comorbidities. Among these comorbidities, obesity stands out, thus highlighting the importance of knowing aspects related to virus infection, especially its association with this health problem. Review: this is an integrative literature review, using databases such as LILACS, MEDLINE, SciELO and PUBMED, searched through the solutions of the following descriptors in Health Sciences (DeCS) and their correspondents in the Medical Subject Headings (Mesh): Comorbidity. Risk factors. Coronavirus infections. Obesity. Discussion: 32 articles were arranged in the light of the literature, demonstrating the relationship between obesity and SARS-CoV-2 infection. Final remarks: With the study's findings, it was possible to understand that obesity presents itself as a risk factor for severe COVID-19, these factors imply important complications and repercussions of the person with obesity: mechanical ventilation, hospitalization and intensive care
\end{abstract}

Keywords: comorbidity, risk factors, coronavirus infections, obesity.

\section{Introdução}

No final do ano de 2019, na cidade de Wuhan, foram relatados diversos casos de indivíduos que estavam desenvolvendo sintomas compatíveis à uma síndrome gripal, a qual precedia episódios de pneumonia, de etiologia desconhecida. Inicialmente, tal surto foi relacionado a um campo geográfico específico, o mercado de frutos do mar, na província de Hubei, na China central. Em dezembro de 2019, identificou-se que essas pessoas estavam infectadas por um novo tipo de coronavírus, nomeado de SARS-CoV-2, responsável pela doença COVID-19 (Song et al., 2020).

Sabe-se que a transmissão se dá através de gotículas oriundas das interações humanas, apresentando um período de incubação que pode variar de 2 a 14 dias. As manifestações clínicas são amplas, em que compreende casos assintomáticos, sintomas gripais e quadros de pneumonia com diferentes graus de gravidade. É oportuno destacar que $80 \%$ dos casos evolui com bom prognóstico e apresentam sintomas que se assemelham 
a um resfriado comum, como cefaleia, tosse, dispneia e febre. Contudo, alguns podem desencadear a forma grave, sendo necessário cuidados intensivos, a exemplo dos idosos ou daqueles que possuem alguma comorbidade (Xavier et al., 2009).

Dentre essas comorbidades, destaca-se a obesidade, definida pela como um acúmulo anormal ou excessivo de gordura que apresenta risco à saúde, apresentando um índice de massa corporal (IMC) acima de 30. Ocorre um desequilíbrio energético entre as calorias consumidas e as calorias gastas. A prevalência mundial de obesidade quase triplicou entre 1975 e 2016 (OMS, 2018). A nível nacional, o Sistema de Vigilância Nutricional (SISVAN) consolidou os dados do ano de 2019 e identificou que cerca de $28,49 \%$ da população adulta brasileira apresentou quadro de obesidade, que variou do grau I ao III (BRASIL, 2019).

Ainda no ano de 2019, o Ministério da Saúde traçou um perfil de obesidade no Brasil através de uma pesquisa de Vigilância de Fatores de Risco e Proteção para Doenças Crônicas por Inquérito Telefônico (VIGITEL), em que foram entrevistados 52.443 pessoas maiores de 18 anos de todas as capitais brasileiras e Distrito Federal. A partir da referida investigação, identificou-se que a prevalência da obesidade passou de 11,8\% no ano de 2016 para 20,3\% em 2019, ou seja, 2 em cada 10 brasileiros estão obesos (BRASIL, 2019).

A relação entre a obesidade e o novo coronavírus ainda estão sendo elucidados. Estudo de coorte retrospectivo realizado em um hospital francês demonstrou que de 124 pacientes que foram admitidos na unidade de terapia intensiva por SARS-CoV-2, 85 necessitavam de Ventilação Mecânica Invasiva (VMI) e cerca de $85,7 \%$ destes, tinham um índice de massa corporal maior que $35 \mathrm{~kg} / \mathrm{m}^{2}$, apresentando mediana de 31,1 $\mathrm{kg} / \mathrm{m}^{2}$, diferindo acentuadamente dos 39 pacientes do grupo controle com mediana de $27.0 \mathrm{~kg} / \mathrm{m}^{2}$ que não precisavam de VMI. Esses dados revelaram uma associação de gravidade entre a obesidade e a infecção pelo coronavírus (Simonnet et al., 2020).

Corroborando isso, o boletim epidemiológico publicado em outubro de 2020, pelo Ministério da Saúde do Brasil, contabilizou 142.783 pessoas que evoluíram para óbito em decorrência da COVID-19, dentre os quais o número de pessoas que tinham obesidade como uma comorbidade como fator agravantes variou entre 3.917 para aqueles maiores ou iguais 60 anos de idade e 3.419 nos indivíduos com menos de 60 anos (BRASIL, 2020).

Considerando as ponderações ora expostas, ressalta-se a importância de conhecer os aspectos relacionados à infecção pelo novo coronavírus, sobretudo sua associação com outros agravos à saúde, a exemplo da obesidade, com vistas a sumarizar os achados baseados em evidências que fundamentam uma melhor compreensão das alterações que ocorrem no organismo do indivíduo e como a obesidade é capaz de agravar o curso da referida infecção. Neste contexto, o presente estudo objetiva identificar, à luz da literatura, a relação entre obesidade e a infecção pelo SARS-CoV-2.

\section{Revisão}

Trata-se de uma revisão integrativa da literatura que será desenvolvido em seis fases, sendo elas: elaboração da questão de investigação; busca na literatura; coleta de dados; análise dos estudos; discussão e apresentação dos estudos incluídos na revisão (Souza et al., 2010).

Como fio condutor desta revisão, elaborou-se o seguinte questionamento: quais os aspectos relacionados entre a obesidade e a infecção pelo SARS-CoV-2? As bases de dados utilizadas foram: Literatura LatinoAmericana e do Caribe em Ciências de Saúde (LILACS), Medical Literature Analysis and Retrieval System Online (MEDLINE), Scientific Electronic Library Online (SciELO) e PUBMED. Para tanto, foram utilizados os seguintes Descritores em Ciências da Saúde (DeCS) e seus correspondentes no Medical Subject Headings (Mesh): coronavirus infections, obesity, comorbidity e risk factors.

Os descritores foram aplicados conjuntamente através do operador booleano "AND” gerando 2 tipos de cruzamentos, sendo o primeiro formado pelos descritores coronavirus infections AND obesity AND comorbidity AND risk factors e o segundo cruzamento pelos descritores coronavirus infections AND obesity AND risk factors.

Foram utilizados os seguintes filtros que serviram como critérios de inclusão dos artigos: artigos em inglês, espanhol e português; textos completos e materiais produzidos sobre a temática nos últimos cinco anos, que estivessem disponíveis na íntegra. Foram excluídos os artigos em outras línguas estrangeiras não incluídas, textos incompletos e que não estavam disponíveis gratuitamente, cartas ao editor, artigos de opinião, e aqueles que não tinha uma metodologia formulada, além de títulos e resumos que não se encontravam nas necessidades e delimitação da temática pesquisada.

Ao ser realizada a busca nas referidas bases de dados, através da primeira aplicação dos critérios de inclusão e exclusão, a pesquisa resultou em 322 artigos, sendo: 2 da SciELO, 66 da MEDLINE, 5 da LILACS e um total de 249 artigos da PUBMED. Após a leitura de títulos e resumos, a nova filtragem permitiu reduzir 
para 113 artigos, sendo: 1 da SciELO, 14 da MEDLINE, 1 da LILACS e 97 da PUBMED. Com a realização da leitura dos resumos foram totalizados 89 artigos, sendo: 1 da SciELO, 14 da MEDLINE, 1 da LILACS e 73 da PUBMED. E por fim, com a leitura na íntegra de todos os estudos, a amostra final foi composta por 32 artigos, sendo 2 da MEDLINE e 30 PUBMED, conforme demonstra a figura 1.

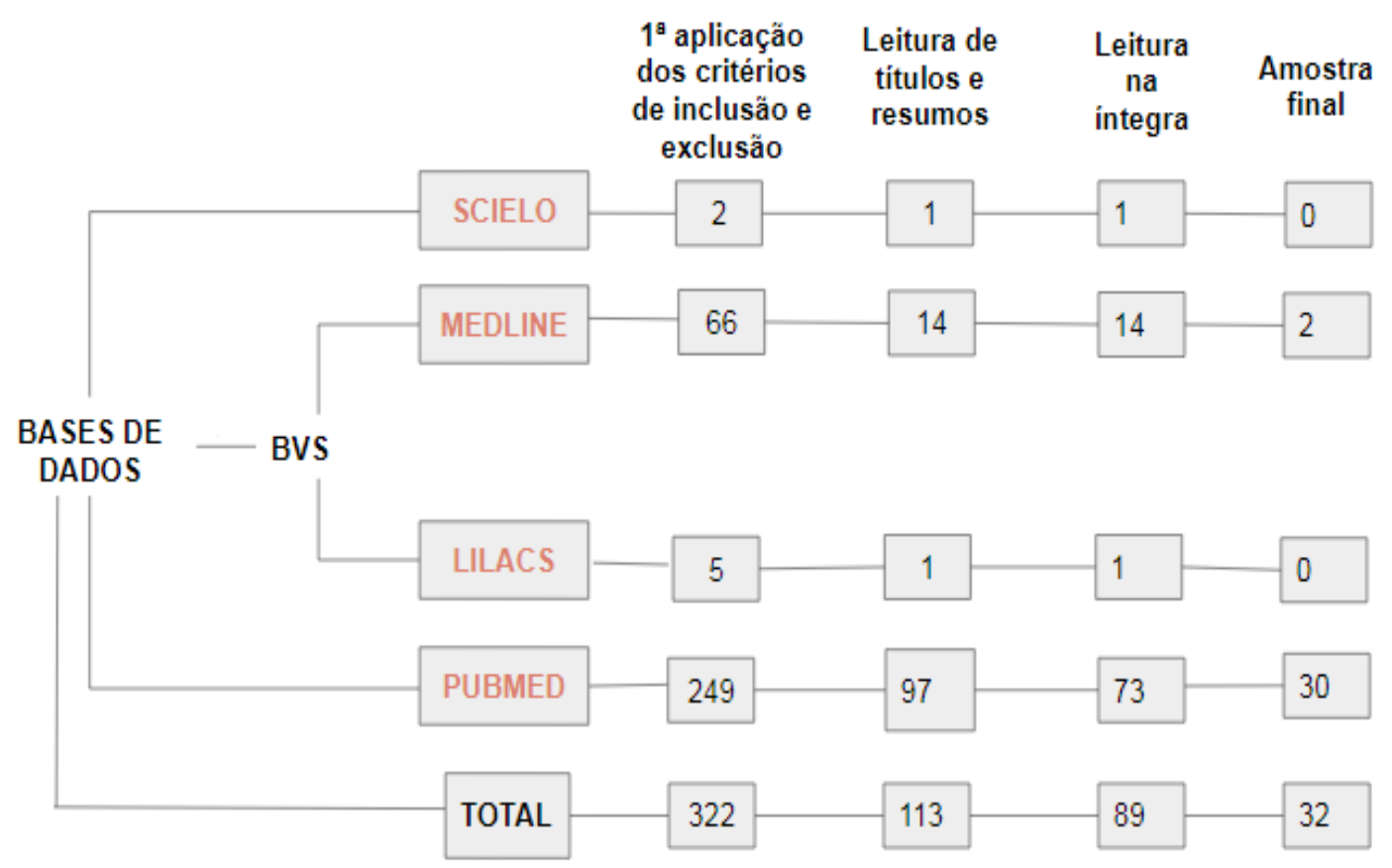

Figura 1. Fluxograma de identificação dos estudos incluídos de acordo com os critérios de elegibilidade pré-estabelecidos. Fonte: Dados da pesquisa, 2020.

Na etapa de coleta dos dados, foi utilizado o instrumento de coleta de dados validado por Ursi (2012). Para análise dos dados, e posterior efetivação das etapas de discussão e apresentação dos estudos incluídos na revisão, utilizou-se análise categorial temática proposta por Bardin, que é realizada por meio das etapas de pré-análise, exploração do material ou codificação e tratamento dos resultados obtidos e interpretação destes (Bardin, 2011).

Destaca-se, ainda, que para elaboração dessa pesquisa, utilizou-se o sistema de classificação composto por sete níveis, sendo: Nível I - evidências oriundas de revisões sistemáticas ou metanálise de relevantes ensaios clínicos; Nível II - evidências derivadas de pelo menos um ensaio clínico randomizado controlado e bem delineado; Nível III - ensaios clínicos bem delineados sem randomização; Nível IV - estudos de coorte e caso controle; Nível V - revisão sistemática de estudos descritivos e qualitativos; Nível VI - evidências derivadas de um único estudo descritivo ou qualitativo; e Nível VII - opinião de autoridades ou relatório de comitês de especialistas (Galvão, 2006). A presente investigação respeitou, ainda, os preceitos éticos, conforme os aspectos que envolvem estudos de revisão integrativa, de maneira que todos autores foram devidamente referenciados e citados.

Todos os artigos selecionados foram publicados no ano de 2020, sendo esse fator justificado pelo conteúdo dos mesmos abordarem em sua literatura o contexto atual da pandemia pelo novo coronavírus. Os países que tiveram prevalência quanto às publicações foram Itália e França com 9,3\%,China com 12,5\% e Estados Unidos da América (EUA) com 31,2\% dos estudos publicados.

Esses países ganharam bastante destaque no que diz respeito às publicações, devido ao crescente número de casos de COVID-19 em suas regiões, sendo a região asiática o primeiro epicentro da doença, em seguida países da Europa e logo após os EUA (Lima et al., 2020). O relatório situacional publicado em dezembro de 2020 pela Organização mundial da saúde mostra no ranking de casos acumulados tendo os EUA em seu primeiro lugar, no segundo lugar a Índia e o Brasil na terceira posição (BRASIL, 2020). Os artigos foram publicados em duas áreas, sendo $90,6 \%$ na área médica e 9,3\% na área de biomedicina, e todos foram publicados no idioma da língua inglesa.

Como critério de definição de relevância dos artigos foram analisados o nível de evidência científica dos estudos publicados prevalecendo cerca de 40,6\% do nível 4, que são provenientes de estudos de coorte e de caso-controle bem delineados; $25 \%$ do nível 5 , originadas de revisão sistemática de estudos descritivos e 
qualitativos e 15,6\% do nível 6, derivadas de um único estudo descritivo ou qualitativo (Galvão, 2006). Na tabela 1 segue a sumarização dos dados.

Tabela 1. Sumarização dos dados em número e porcentagem das bases de dados, níveis de evidência, local de publicação, idioma e área de publicação dos artigos selecionados.

\begin{tabular}{|c|c|c|}
\hline Base de dados & $\mathrm{N}^{\mathrm{o}}$ & $\%$ \\
\hline Pubmed & 2 & $6,25 \%$ \\
\hline Medline & 30 & $93,75 \%$ \\
\hline \multicolumn{3}{|c|}{ Nível de evidência } \\
\hline Nível 1 & 3 & 9,375 \\
\hline Nível 3 & 3 & 9,375 \\
\hline Nível 4 & 13 & 40,625 \\
\hline Nível 5 & 8 & 25 \\
\hline Nível 6 & 5 & 15,625 \\
\hline \multicolumn{3}{|c|}{ Local de Publicação } \\
\hline Alemanha & 1 & 3,125 \\
\hline Brasil & 3 & 9,375 \\
\hline China & 4 & 12,5 \\
\hline EUA & 10 & 31,25 \\
\hline França & 3 & 9,375 \\
\hline Grécia & 1 & 3,125 \\
\hline Hungria & 1 & 3,125 \\
\hline Îndia & 1 & 3,125 \\
\hline Itália & 3 & 9,375 \\
\hline México & 2 & 6,25 \\
\hline Suíça & 1 & 3,125 \\
\hline Reino Unido & 1 & 3,125 \\
\hline \multicolumn{3}{|l|}{ Idioma } \\
\hline Inglês & 32 & 100 \\
\hline \multicolumn{3}{|c|}{ Área de publicação } \\
\hline Médica & 29 & 90,625 \\
\hline Biomédica & 3 & 9,375 \\
\hline
\end{tabular}

No processo de análise dos dados foram delimitadas unidades de registro, que posteriormente, foram tematicamente ordenadas em duas categorias relativas à: fisiopatologia da obesidade associada à infecção do Sars-Cov-2 e desfechos de saúde da interação obesidade com a COVID-19, visando o melhor entendimento do fenômeno e o alcance do objetivo desta revisão.

\section{Discussão}

\section{Fisiopatologia da obesidade associada à infecção do Sars-Cov-2}

No século XXI a obesidade é encarada como uma crise global, devido a diversas associações de impacto negativo, como Diabetes Melittus, hipertensão e outros distúrbios cardiovasculares, e atualmente efeitos de gravidade na progressão do quadro da COVID-19, devido à relação com essas morbidades e suas implicações fisiopatológicas. A obesidade em si, já se caracteriza como um potencial fator de risco no do curso dessa doença, por conta dos fatores mecânicos, imunológicos e fisiológicos alterados (Petrova et al., 2020).

O tecido adiposo encontrado em grande quantidade nos indivíduos obesos também é responsável pelo equilíbrio hemostático e hemodinâmico do corpo, um bom exemplo disso é a proteína hepcidina que é identificada em quantidades demasiadas quando o índice de massa corporal humana é elevado. Ela está 
diretamente relacionada à hipóxia e diminuição da oxigenação tecidual, afetando a hemostasia do ferro e levando a inflamações crônicas (Silva et al., 2019).

A obesidade pode ser considerada como critério de gravidade em pacientes acometidos pelo novo coronavírus devido à diminuição da capacidade ventilatória frente ao grande aporte de massa gordurosa no abdome e tórax. Além das alterações mecânicas evidenciadas, há uma resposta inflamatória exacerbada chamada tempestade de citocinas. Destaca-se que, as células de gordura estão distribuídas em todo corpo e comumente se acumulam no abdômen, essas mesmas células liberam adipocinas, que tem a função semelhante às citocinas, desencadeando reações linfocitárias em larga escala (Beretta, 2020).

A dipeptidilpeptidase (DPP-4) é uma adipocina pró-inflamatória liberada pelo tecido adiposo que está relacionada a resistência à insulina, à inflamação de baixo grau e ainda em fase de estudo, acredita-se que essa substância também pode participar na internalização do vírus na célula e quando circulante em altas quantidades no sangue pode ser de potencial importância para complicações metabólicas agudas de longo prazo da COVID-19 (Schilicht et al., 2020).

As respostas causam reações inflamatórias crônicas que, em longo prazo, danificam os tecidos do corpo e podem desencadear outros processos de adoecimento. Da mesma maneira, ocorre o processo inflamatório quando as células dos alvéolos são infectadas pelo vírus e, o que poderia ser uma resposta inflamatória normal, ganha grande nível de intensidade quando combinadas, acarretando em prejuízos para o funcionamento normal do corpo (Berreta, 2020).

Os pacientes obesos comumente desenvolvem dificuldades em sua imunidade no enfrentamento de outras infecções, podendo levá-los a quadros de sepse e consequentemente evoluir para óbito (Brandão et al., 2020). Pesquisas apontam que pessoas com hipertensão, obesidade, e diabetes foram mais propensas a serem hospitalizadas do que pessoas sem essas comorbidades (Carrilo-Vega et al., 2020). Um estudo de coorte realizado em Nova York com pacientes COVID-19, constatou que os mesmos com obesidade tinham maior probabilidade de apresentar sintomas evidentes, incluindo febre, tosse e dispneia, além de tornar a doença a nível crítico (Hajifathalian et al., 2020).

Em 2009, o surto da infecção pelo vírus H1N1 apresentou pessoas obesas com quadros de complicações, ou seja, esta comorbidade já foi relacionada como um fator de risco para outras doenças de causa viral, o que desperta um olhar atento. Além do estado de inflamação crônica, o corpo obeso progride para um estado de resposta imunológica retardada, bem como para uma memória imunológica deficiente, apresentando, portanto, uma maior propensão intrínseca à COVID-19 (Costa et al., 2020).

A obesidade pode trazer outros distúrbios e culminar na síndrome metabólica, aliando ao quadro: resistência à insulina, dislipidemia e hipertensão; sendo agravantes para outras comorbidades. A mecânica respiratória se encontra prejudicada, a fisiologia pulmonar restrita devido ao excesso de peso e a reserva pulmonar insuficiente, contribuindo para doenças respiratórias graves; ainda há disfunção endotelial levando a eventos trombóticos aumentados, tudo isso compondo um pior prognóstico se associado à COVID-19 (Frank et al., 2020). Apesar da fisiopatologia do COVID-19 não ser inteiramente esclarecida, sabe-se que o SARSCoV-2 se liga ao receptor da enzima conversora de angiotensina-2 (ECA2). Devido ao alto grau de expressão da ECA2, alguns órgãos se tornam mais vulneráveis à infecção pelo SARS-CoV-2, como os pulmões, coração, esôfago, rins, bexiga e íleo, motivo pelo qual ocorrem as manifestações extrapulmonares (Beeching et al., 2020). Além da deficiência do sistema imune e todos os fatores anteriormente explicitados à respeito da obesidade e sua fisiopatologia, outra explicação para seu agravamento no quadro da COVID-19 está no fato da expressão do ECA2 no tecido adiposo. Aqui, os níveis de expressão do ECA2 excedem até mesmo os níveis expressos nos pulmões, portanto, indivíduos mais obesos apresentam mais tecido adiposo e consequentemente mais níveis de ECA2. Fato este que implicaria maior suscetibilidade à COVID-19 (Silva et al., 2021).

\section{Consequências e desfechos de saúde da interação obesidade com a COVID-19}

A obesidade está relacionada a uma pior resposta imunológica e desfechos em pacientes com doenças respiratórias. Partindo dessa informação, foi feito um estudo que comparou pacientes internados com SARSCoV-2 no hospital de Shenzhen, na China, que apresentavam peso normal, sobrepeso e obesidade. Dentre os achados, foi visto que os pacientes obesos apresentam como sintomas iniciais a febre e tosse; além disso, diante dos dados da amostra, os pacientes obesos têm maior probabilidade de desenvolver COVID-19 grave (Cai et al., 2020).

Em pacientes obesos e com a COVID-19, a interação com o sistema imunológico, como trazido anteriormente, é uma das razões da facilitação da gravidade e letalidade. Com isso, ambas doenças foram caracterizadas como emergências de saúde pública internacional pela OMS (Organização Mundial da Saúde). Os estudos epidemiológicos tiveram, até então, um papel sobressalente nas pesquisas e no estabelecimento 
de condutas e protocolos. Isso porque em pouco tempo conseguiu-se identificar e listar alguns fatores de agravo, a obesidade entre eles, promovendo assim, maior atenção para esse grupo de pacientes (Petrakis et al., 2020).

A associação da obesidade e COVID-19 em estudos têm mostrado consideráveis complicações e repercussões importantes. Em um estudo de coorte, os pacientes hospitalizados confirmados com SARS-CoV2 , que possuíam IMC $\geqslant 30 \mathrm{~kg} / \mathrm{m} 2$ era considerado um fator de risco forte. A maior dificuldade respiratória na entrada hospitalar, inicialmente, com aumento da dispneia e frequência cardíaca, necessidade de oxigenoterapia evoluindo para maiores taxas de intubação. Em 124 pacientes já internados na UTI, o IMC 》 $35 \mathrm{~kg} / \mathrm{m} 2$ foi um fator importante associado à necessidade de ventilação mecânica. Outros estudos americanos apontam que o IMC $\geqslant 30 \mathrm{~kg} / \mathrm{m} 2$ foi associado a um risco aumentado de hospitalização e cuidados intensivos (Frank et al., 2020).

Entre os pacientes admitidos com COVID-19 na UTI, 40\% a 100\% dos pacientes necessitam de VMI. Ademais, o excesso de massa corpórea e a apneia obstrutiva comprometem muitas vezes o suporte respiratório e intubação endotraqueal. A permanência prolongada na intubação endotraqueal, mais frequente nos doentes obesos, aumenta o risco de infecção da equipe intensivista. Os pacientes que apresentam IMC mais elevados requerem uma VMI mais constantemente e por um período mais longo. Todavia este tempo prolongado em VMI pode acarretar em maiores riscos para os pacientes como contraírem outras infecções, lesões ocasionadas pelo tubo endotraqueal, secreções, afonia e o próprio desconforto proporcionado pela terapêutica relatados por muitos pacientes como sensação de sufocamento (Földi et al., 2020).

Em um outro estudo, baseado numa análise de 265 pacientes, sendo $58 \%$ do sexo masculino, verificou-se que a maioria dos pacientes mais jovens que necessitaram de internação, eram obesos. A mediana do IMC foi de $29,3 \mathrm{~kg} / \mathrm{m} 2$, onde apenas $25 \%$ dos indivíduos apresentaram IMC menor que $26 \mathrm{~kg} / \mathrm{m} 2$ e $25 \%$ deles, com um IMC superior a $34,7 \mathrm{~kg} / \mathrm{m} 2$; os $50 \%$ restantes, se mantiveram numa faixa de sobrepeso e obesidade grau 1 . Percebe-se, com isso, uma inversão no que se observava com internações majoritariamente daqueles com idade mais avançada, agora com jovens obesos internados numa proporção mais próxima (Kass et al., 2020).

Tanto a hiperinflamação como a coagulação têm sido relatadas como uma constatação frequente em ambas as COVID-19 e obesidade. Estudos levantam a hipótese de que a obesidade está alterando a resposta imunitária, de modo que as funções e proteções imunológicas podem estar desativadas, associando assim que o sucesso da vacinação pode ser questionável nas pessoas obesas, sendo importante a realização de novas pesquisas a partir desse fato (Földi et al., 2020).

A obesidade, nos relatórios clínicos iniciais, foi pouco mencionada quanto aos fatores de risco relacionados à infecção pelo SARS-CoV-2. No entanto, como foi visto ao longo de diversas pesquisas, os novos dados reforçam a alta frequência da obesidade entre os pacientes admitidos nas Unidades de Terapia Intensiva. Ainda, segundo KASS et al (2020) a obesidade também pode restringir a ventilação, por agir impedindo ou dificultando a excursão do diafragma, além de ser pró-inflamatória, induzir o estresse oxidativo e diabetes. Por ter uma correlação considerável em termos de metabolismo com o diabetes mellitus, a obesidade e a grande quantidade gordura ectópica estão também associadas à resistência à insulina. Tal fato fortalece ainda, a conexão com o diabetes como outra comorbidade estreitamente identificada em diferentes estudos (Sattar et al., 2020).

Numa perspectiva de resposta cardiovascular, testes e indicativos genéticos demonstram que a obesidade está relacionada à hipertensão, doença cardíaca coronária, derrame, fibrilação atrial, doença renal, insuficiência cardíaca e aumento do risco de trombose. Importante salientar tais dados, visto que muitas dessas complicações também são associadas a distúrbios graves da COVID-19, como coagulação intravascular disseminada pró-trombótica e tromboembolismo venoso (Sattar et al., 2020).

\section{Considerações finais}

Com os achados do estudo foi possível compreender que a obesidade se apresenta como um fator de risco para a COVID-19 grave. A obesidade implica em um corpo com reações inflamatórias crônicas, fatores mecânicos, imunológicos e fisiológicos comprometidos. Além disso, a obesidade geralmente vem associada a outras doenças que também estão envolvidas em complicações na COVID-19.

Ainda, sabe-se que o SARS-CoV2 (ECA2) se liga ao receptor da enzima conversora de angiotensina 2, fato este que se associa ao alto grau de expressão da ECA2 no tecido adiposo. Todos esses fatores implicam em complicações e repercussões importantes da pessoa com obesidade: ventilação mecânica, hospitalização e cuidados intensivos. 
Sabendo da gravidade da obesidade junto à COVID-19, é necessário um olhar atento para estes pacientes, dando o devido valor na sua apresentação. Por fim, este artigo serve como embasamento para pesquisas epidemiológicas e subsídios para os profissionais de saúde para realizar a prática de evidências científicas.

\section{Agradecimentos}

Agradecemos ao Núcleo de Estudos e Pesquisas em Saúde do Adulto e do Idoso (NEPSAI) pelo incentivo na produção deste artigo.

\section{Referência}

Barding, L. Análise de conteúdo. 2011. São Paulo: Edições 70, 229

Beeching, N.J., Fletcher, T.E., Fowler, R. 2020. Doença do coronavírus. BMJ, Best Pract.

Beretta, A. 2020. Obesity, inflammation and COVID-19. Swiss Med Wkly, 150(33-34), 2-3.

Brandão, S., Godoi, E., Cordeiro, L., Bezerra, C., Ramos, J., Arruda, G. 2020. Obesidade e Risco de COVID-19 Grave. Repositório Digital da UFPE.

Brasil. Ministério da Saúde. Secretaria de Vigilância em Saúde. Departamento de Análise em Saúde e Vigilância de Doenças Não Transmissíveis. Vigitel Brasil 2019: Vigilância de fatores de risco e proteção para doenças crônicas por inquérito telefônico: Estimativas sobre frequência e distribuição sociodemográfica de fatores de risco e proteção para doenças crônicas nas capitais dos 26 estados brasileiros e no Distrito Federal em 2019.

Brasil. Ministério da Saúde - Secretaria de Vigilância em Saúde. 2020. Boletim epidemiológico especial: Doença pelo Coronavírus COVID-19.

Cai, Q., Chen, F., Wang, T., Luo, F., Liu, X., Wu, Q., 2020. Obesity and COVID-19 Severity in a Designated Hospital in Shenzhen, China. Diabetes Care, 43(7),1392-8.

Carrillo-Vega, M.F., Salinas-Escudero, G., García-Peña, C., Gutiérrez-Robledo, L.M., Parra-Rodríguez, L. 2020. Early estimation of the risk factors for hospitalization and mortality by COVID-19 in Mexico. PLoS One,15,1-11.

Costa, F.F., Rosário, W.R., Ribeiro, F.A.C., Souza, R.G., Duarte, G.R.S., Barroso, W.A. 2020. Metabolic syndrome and COVID-19: An update on the associated comorbidities and proposed therapies. Diabetes Metab Syndr, 14(5), 809-814.

Földi, M., Farkas, N., Kiss, S., Zádori, N., Váncsa, S., Szakó, L. 2020. Obesity is a risk factor for developing critical condition in COVID-19 patients: A systematic review and meta-analysis. Obes Rev, 21(10),1-9.

Frank, R.C., Mendez, S.R., Stevenson, E.K., Guseh, J.S., Chung, M., Silverman, M.G. 2020. Obesity and the risk of intubation or death in patients with coronavirus disease 2019. Crit Care Med, 1097-101.

Galvão, C.M. Níveis de evidência. 2006. ACTA Paul Enferm,19(2).

Hajifathalian, K., Kumar, S., Newberry, C., Shah, S., Fortune, B., Krisko, T. 2020. Obesity is Associated with Worse Outcomes in COVID-19: Analysis of Early Data from New York City. Obesity, 28(9):1606-12.

Kass, D.A., Duggal, P., Cingolani, O. 2020. Obesity could shift severe COVID-19 disease to younger ages. Lancet. Elsevier Ltd, 395(10236),1544-5.

Lima, N.T., Buss, P.M., Paes-Sousa R. 2020. COVID-19 pandemic: A health and humanitarian crisis. Cad Saude Publica, 36(7).

Petrakis, D., Margină, D., Tsarouhas, K., Tekos, F., Stan, M., Nikitovic, D. 2020. Obesity - a risk factor for increased COVID-19 prevalence, severity and lethality (Review). Mol Med Rep,22(1):9-19.

Petrova, D., Salamanca-Fernández, E., Rodríguez, B. M., Navarro, P.P., Jiménez, M.J.J., Sánchez, M.J. 2020. Obesity as a risk factor in COVID-19: Possible mechanisms and implications. Aten Primaria, 52(7):496-500.

Sattar, N., McInnes, I.B., McMurray, J.J.V. 2020. Obesity Is a Risk Factor for Severe COVID-19 Infection: Multiple Potential Mechanisms. Circulation, 4-6.

Schlicht, K., Rohmann, N., Geisler, C., Hollstein,T., Knappe, C., Hartmann, K. 2020. Circulating levels of soluble Dipeptidylpeptidase-4 are reduced in human subjects hospitalized for severe COVID-19 infections. Int J Obes, 44(11):2335-8.

Silva, NI.,. Sobrinho, H.M.R., Blanch, G.T., Cruvinel, W.M., Gomes, C.M. 2019.Adipocinas e sua relação com a obesidade. Rev Ciências Ambient e Saúde, 46(1). 
Silva, R.B., Santos, T.M., Araújo, R.M.S., Albuquerque, L.P.2021. Por que a obesidade é um fator agravante para a COVID-19? / Why is obesity an aggravating factor for COVID-19? Brazilian J Heal Rev, 4(2):6502-17.

Simonnet, A., Chetboun, M., Poissy, J., Raverdy, V., Noulette, J., Duhamel, A., Verkindt, H. 2020. High Prevalence of Obesity in Severe Acute Respiratory Syndrome Coronavirus-2 (SARS-CoV-2) Requiring Invasive Mechanical Ventilation. Obesity, 28(7), 1195-1199.

Song, Y., Zhang, M., Yin, L., Wang, K., Zhou, Y., Zhou, M., Lu, Y. 2020. COVID-19 treatment: close to a cure? A rapid review of pharmacotherapies for the novel coronavirus (SARS-CoV-2). Int J Infect Dis, 56(2):106080.

Souza, M. T. S., Silva, M.D., Carvalho R. Revisão Integrativa: o que é e como fazer? 2010. Einstein, 8(1)

Xavier, A.R., Silva, J.S., Almeida, J.P.C.L., Conceição, J.F.E., Lacerda, G.S., Kanaan, S. COVID-19: manifestações clínicas e laboratoriais na infecção pelo novo coronavírus. 2020. J Bras Patol e Med Lab, 56(5):1-9.

World Health Organisation. 2018. Obesity-and-Overweight. https://www.who.int/healthtopics/obesity\#tab=tab_1. p. 1 .

\section{Minicurrículo}

Bruna Leite Lourenço. Graduada em Arquitetura e Graduanda em Medicina pelo Centro Universitário de João Pessoa- UNIPÊ. Participou dos projetos: Núcleo de Pesquisa em Saúde do Adulto e do Idoso - NEPSAI; Dor como $5^{\circ}$ sinal vital; Acompanhamento ambulatorial do uso terapêutico da Cannabis sativa e Projeto Educação em Saúde da criança e do adolescente.

Fabiana Maria Rodrigues Lopes de Oliveira. Doutora em Enfermagem pelo Programa de Pós-Graduação em Enfermagem (PPGEnf) da Universidade Federal da Paraíba (UFPB). Mestre em Enfermagem pelo PPGEnf/UFPB. Pós-Graduada em Saúde das Famílias e das Comunidades pela Universidade Federal de Pernambuco (UFPE). Pós-Graduada em Enfermagem do Trabalho pela Especializa Saúde. Bacharela e Licenciada em Enfermagem pela UFPB. Pesquisadora na área de saúde do idoso. Participante do Grupo de Estudo e Pesquisa em Saúde do Adulto e do Idoso (NEPSAI - UFPB/CNPQ).

Mariana Guedes Almeida. Graduada em Fisioterapia pelo Centro Universitário de João Pessoa - UNIPÊ. Participou do Núcleo de Pesquisa em Saúde do Adulto e do Idoso - NEPSAI. Atuou como extensionista no Projeto Programa de Reabilitação Pós COVID-19 e Projeto Funcionalidade na UTI. Formação complementar no Curso Teórico-Prático: Intervenção Fisioterapêutica na COVID-19 - Da UTI ao ambiente domiciliar.

Renata de Aquino Ferreira. Graduanda em Medicina pelo Centro Universitário de João Pessoa- UNIPÊ. Instrutora de Yoga pelo Dhyana Yoga - João Pessoa (PB). Massoterapeuta Ayurvédica pelo VEDA - Centro de Terapias Integrativas - João Pessoa (PB). Atuou como ligante na Liga Acadêmica de Medicina de Família e Comunidade da Paraíba - LAMFAC-PB. Participou do Núcleo de Pesquisa em Saúde do Adulto e do Idoso NEPSAI.

Sarah Emilly Ramos de Sousa. Graduada em Enfermagem pelo Centro Universitário de João Pessoa- UNIPÊ. Terapeuta Floral. Pós-graduanda em Saúde Pública e Saúde da Família pelo Centro Integrado de Serviços de Consultoria Educacional - CISCE. Participou do Núcleo de Pesquisa em Saúde do Adulto e do Idoso - NEPSAI. Atuou no projeto Unipê Transforma, com ênfase no subprojeto farmacologia descomplicada.

Como citar: Lourenço, B.L., Oliveira, F.M.R.L., Almeida, M.G., Ferreira, R.A., \& Sousa, E.R.S. 2022. Aspectos relacionados a associação entre obesidade e Covid-19: uma revisão integrativa. Pubsaúde, 8, a321. DOI: https://dx.doi.org/10.31533/pubsaude8.a321

Recebido: 7 jan. 2022.

Revisado e aceito: 9 fev. 2022.

Conflito de interesse: os autores declaram, em relação aos produtos e companhias descritos nesse artigo, não ter interesses associativos, comerciais, de propriedade ou financeiros que representem conflito de interesse.

Licenciamento: Este artigo é publicado na modalidade Acesso Aberto sob a licença Creative Commons Atribuição 4.0 (CC-BY 4.0). 